Supporting Information for

\title{
Structure and Photoluminescence Transformation in Hybrid Manganese(II) Chlorides
}

Li Tan, ${ }^{+}$Zhishan Luo, ${ }^{+}$Xiaoyong Chang, Yi Wei, Min Tang, Wen Chen, Qian Li, Pengfei Shen, Zewei Quan*

Department of Chemistry, Academy for Advanced Interdisciplinary Studies, Shenzhen Engineering Research Center for Frontier Materials Synthesis at High Pressures, Southern University of Science and Technology (SUSTech), Shenzhen, Guangdong 518055, China 


\section{Table of Content}

Table S1. Single crystal X-ray data............................................

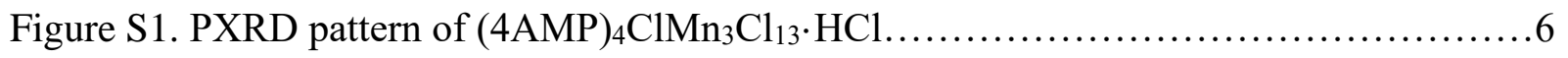

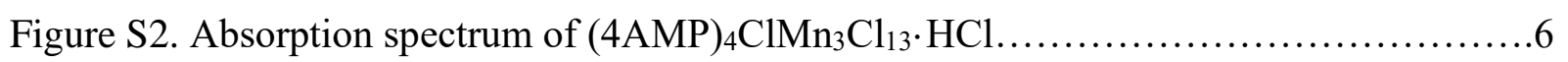

Figure S3. PLE spectra of $(4 \mathrm{AMP})_{4} \mathrm{ClMn}_{3} \mathrm{Cl}_{13} \cdot \mathrm{HCl}$ monitored at different wavelengths........7

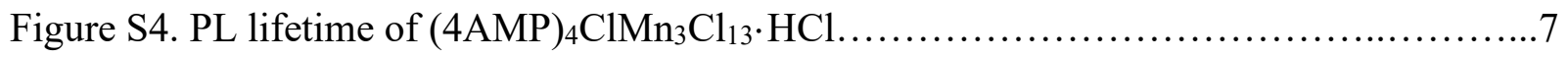

Figure S5. Temperature-dependent PL spectra of $(4 \mathrm{AMP}){ }_{4} \mathrm{ClMn}_{3} \mathrm{Cl}_{13} \cdot \mathrm{HCl} \ldots \ldots \ldots \ldots \ldots \ldots$

Figure S6. Photographs of compounds under $365 \mathrm{~nm}$ UV light........................

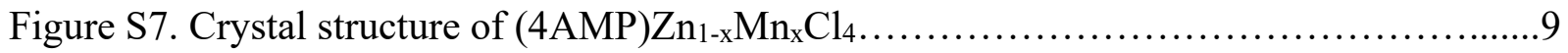

Figure S8. Crystal structures of 1D and OD metal halides..............................9

Figure S9. PXRD patterns of (4AMP) $\mathrm{Zn}_{0.85} \mathrm{Mn}_{0.15} \mathrm{Cl}_{4}$ and $(4 \mathrm{AMP}) \mathrm{ZnCl}_{4} \ldots \ldots \ldots \ldots \ldots \ldots . . . \ldots$

Table S2. Measured compositions of the as-prepared single crystals by ICP-MS..............11

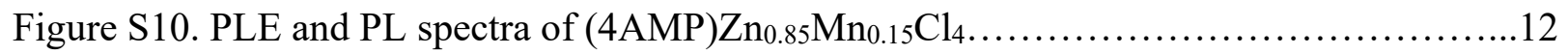

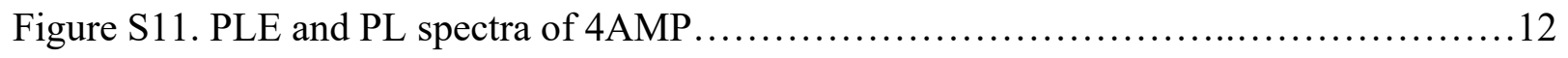

Figure S12. Photographs of the formation of mixture of 1D and OD metal halides at a low Zn:Mn

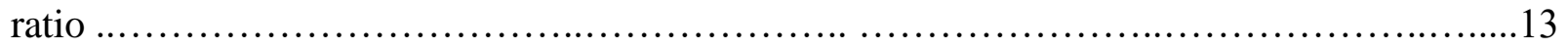

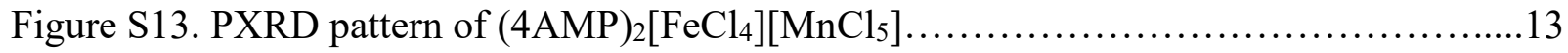

Figure $\mathrm{S} 14 . \mathrm{PXRD}$ pattern of $(4 \mathrm{AMP})_{4} \mathrm{ClCo}_{0.21} \mathrm{Mn}_{2.79} \mathrm{Cl}_{13} \cdot \mathrm{HCl} \ldots \ldots \ldots \ldots \ldots \ldots \ldots \ldots \ldots \ldots . \ldots \ldots$

Figure $\mathrm{S} 15 . \mathrm{PXRD}$ pattern of $(4 \mathrm{AMP})_{4} \mathrm{ClNi}_{0.06} \mathrm{Mn}_{2.94} \mathrm{Cl}_{13} \cdot \mathrm{HCl} \ldots \ldots \ldots \ldots \ldots \ldots \ldots \ldots \ldots \ldots 14$

Figure $\mathrm{S} 16 . \mathrm{PXRD}$ pattern of $(4 \mathrm{AMP})_{4} \mathrm{ClCu}_{2.4} \mathrm{Mn}_{0.6} \mathrm{Cl}_{13} \cdot 2 \mathrm{HCl} \ldots \ldots \ldots \ldots \ldots \ldots \ldots \ldots \ldots \ldots$

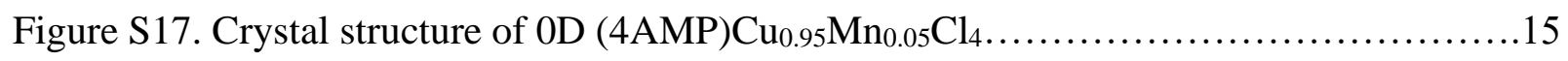


Figure S18. Photographs of the obtained single crystals under natural light............... 16

Figure S19. PLE spectrum of (4AMP) ${ }_{4} \mathrm{ClNi}_{0.06} \mathrm{Mn}_{2.94} \mathrm{Cl}_{13} \cdot \mathrm{HCl}$ monitored at $630 \mathrm{~nm} . \ldots \ldots . .16$

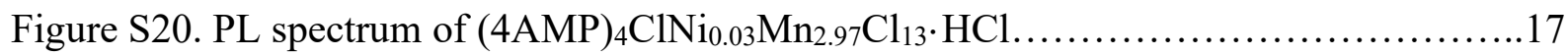


Table S1. Single crystal X-ray data of $(4 \mathrm{AMP})_{4} \mathrm{ClMn}_{3} \mathrm{Cl}_{13} \cdot \mathrm{HCl}$, (4AMP) $\mathrm{Zn}_{0.85} \mathrm{Mn}_{0.15} \mathrm{Cl}_{4}$ and (4AMP) $\mathrm{ZnCl}_{4}$.

\begin{tabular}{|c|c|c|c|}
\hline Empirical Formula & $\begin{array}{c}(4 \mathrm{AMP})_{4} \mathrm{ClMn}_{3} \mathrm{Cl}_{13} \cdot \\
\mathrm{HCl}\end{array}$ & $\begin{array}{c}(4 \mathrm{AMP}) \mathrm{Zn}_{0.85} \mathrm{Mn}_{0 .} \mathrm{Cl}_{4} \\
{ }_{15}\end{array}$ & $(4 \mathrm{AMP}) \mathrm{ZnCl}_{4}$ \\
\hline Formula weight & 1137.21 & 333.80 & 335.35 \\
\hline Temperature/K & 100.0 & 100.0 & 100.0 \\
\hline Crystal system & orthorhombic & monoclinic & monoclinic \\
\hline Space group & Pmmn & $P 2_{1}$ & $P 2_{1}$ \\
\hline $\mathrm{a} / \AA$ & $17.4146(8)$ & $6.1020(3)$ & $6.1095(5)$ \\
\hline $\mathrm{b} / \AA \AA$ & $24.6624(11)$ & $13.3200(7)$ & $13.2981(11)$ \\
\hline $\mathrm{c} / \AA$ & $5.1803(2)$ & $7.4894(4)$ & $7.4741(6)$ \\
\hline$\alpha /{ }^{\circ}$ & 90 & 90 & 90 \\
\hline$\beta /{ }^{\circ}$ & 90 & $99.108(2)$ & $99.108(2)$ \\
\hline$\gamma /{ }^{\circ}$ & 90 & 90 & 90 \\
\hline Volume $/ \AA^{3}$ & $2224.87(17)$ & $601.05(5)$ & $599.58(8)$ \\
\hline $\mathrm{Z}$ & 2 & 2 & 2 \\
\hline$\rho_{\text {calc }} \mathrm{g} / \mathrm{cm}^{3}$ & 1.698 & 1.844 & 1.857 \\
\hline$\mu / \mathrm{mm}^{-1}$ & 1.770 & 2.760 & 2.909 \\
\hline $\mathrm{F}(000)$ & 1140.0 & 334.5 & 336.0 \\
\hline Crystal size/mm3 & $0.1 \times 0.05 \times 0.05$ & $0.4 \times 0.2 \times 0.1$ & $0.1 \times 0.1 \times 0.1$ \\
\hline Radiation & $\operatorname{MoK} \alpha(\lambda=0.71073)$ & $\begin{array}{c}\operatorname{MoK} \alpha(\lambda= \\
0.71073)\end{array}$ & $\begin{array}{c}\operatorname{MoK} \alpha(\lambda= \\
0.71073)\end{array}$ \\
\hline
\end{tabular}




\begin{tabular}{|c|c|c|c|}
\hline $\begin{array}{l}2 \Theta \text { range for data } \\
\text { collection/ } /{ }^{\circ}\end{array}$ & 4.678 to 55.148 & 6.118 to 55.1 & 6.128 to 55.036 \\
\hline Index ranges & $\begin{array}{l}-22 \leq \mathrm{h} \leq 22,-32 \leq \mathrm{k} \\
\leq 32,-6 \leq 1 \leq 6\end{array}$ & $\begin{array}{c}-7 \leq \mathrm{h} \leq 7,-17 \leq \mathrm{k} \\
\leq 17,-9 \leq 1 \leq 9\end{array}$ & $\begin{array}{c}-7 \leq \mathrm{h} \leq 7,-15 \leq \mathrm{k} \\
\leq 15,-8 \leq 1 \leq 8\end{array}$ \\
\hline Reflections collected & 40835 & 8573 & 11281 \\
\hline Independent reflections & $\begin{array}{c}2717\left[\mathrm{R}_{\text {int }}=0.0360\right. \\
\left.\quad \mathrm{R}_{\text {sigma }}=0.0134\right]\end{array}$ & $\begin{array}{c}2745\left[\mathrm{R}_{\text {int }}=\right. \\
0.0289, \mathrm{R}_{\text {sigma }}= \\
0.0399]\end{array}$ & $\begin{array}{c}2092\left[\mathrm{R}_{\text {int }}=\right. \\
0.0529, \mathrm{R}_{\text {sigma }}= \\
0.0413]\end{array}$ \\
\hline $\begin{array}{c}\text { Data/restraints/paramet } \\
\text { ers }\end{array}$ & $2717 / 0 / 134$ & $2745 / 1 / 137$ & $2690 / 1 / 140$ \\
\hline Goodness-of-fit on $\mathrm{F}^{2}$ & 1.076 & 0.971 & 1.047 \\
\hline $\begin{array}{l}\text { Final } \mathrm{R} \text { indexes }[\mathrm{I}>=2 \sigma \\
\text { (I)] }\end{array}$ & $\begin{array}{c}\mathrm{R}_{1}=0.0254, \mathrm{wR}_{2}= \\
0.0657\end{array}$ & $\begin{aligned} \mathrm{R}_{1} & =0.0189, \mathrm{w} \mathrm{R}_{2} \\
& =0.0357\end{aligned}$ & $\begin{aligned} \mathrm{R}_{1}= & 0.0289, \mathrm{wR} \mathrm{R}_{2} \\
& =0.0709\end{aligned}$ \\
\hline $\begin{array}{l}\text { Final } \mathrm{R} \text { indexes [all } \\
\text { data] }\end{array}$ & $\begin{array}{c}\mathrm{R}_{1}=0.0302, \mathrm{w} \mathrm{R}_{2}= \\
0.0684\end{array}$ & $\begin{array}{c}\mathrm{R}_{1}=0.0220, \mathrm{wR}_{2} \\
=0.0364\end{array}$ & $\begin{array}{c}\mathrm{R}_{1}=0.0298, \mathrm{wR} 2 \\
=0.0712\end{array}$ \\
\hline $\begin{array}{c}\text { Largest diff. peak/hole / } \\
\text { e } \AA^{-3}\end{array}$ & $0.38 /-0.51$ & $0.23 /-0.26$ & $1.71 /-0.44$ \\
\hline
\end{tabular}




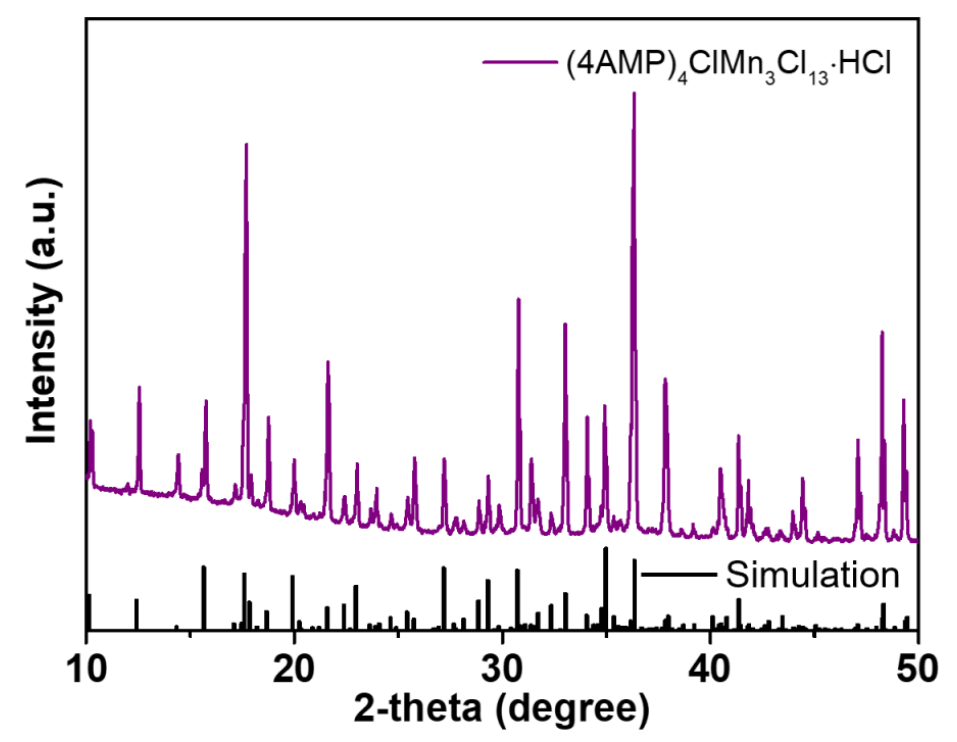

Figure S1. Comparison between the detected and simulated XRD patterns of compound (4AMP) $)_{4} \mathrm{ClMn}_{3} \mathrm{Cl}_{13} \cdot \mathrm{HCl}$. The simulation pattern is extracted from SCXRD.

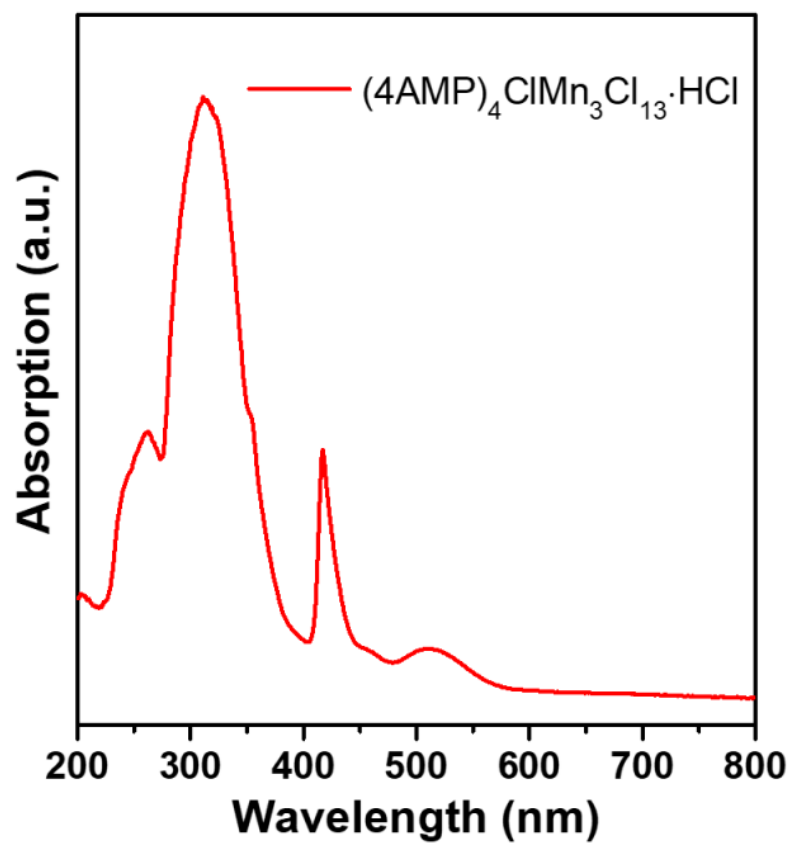

Figure S2. Absorption spectrum of $(4 \mathrm{AMP})_{4} \mathrm{ClMn}_{3} \mathrm{Cl}_{13} \cdot \mathrm{HCl}$. 


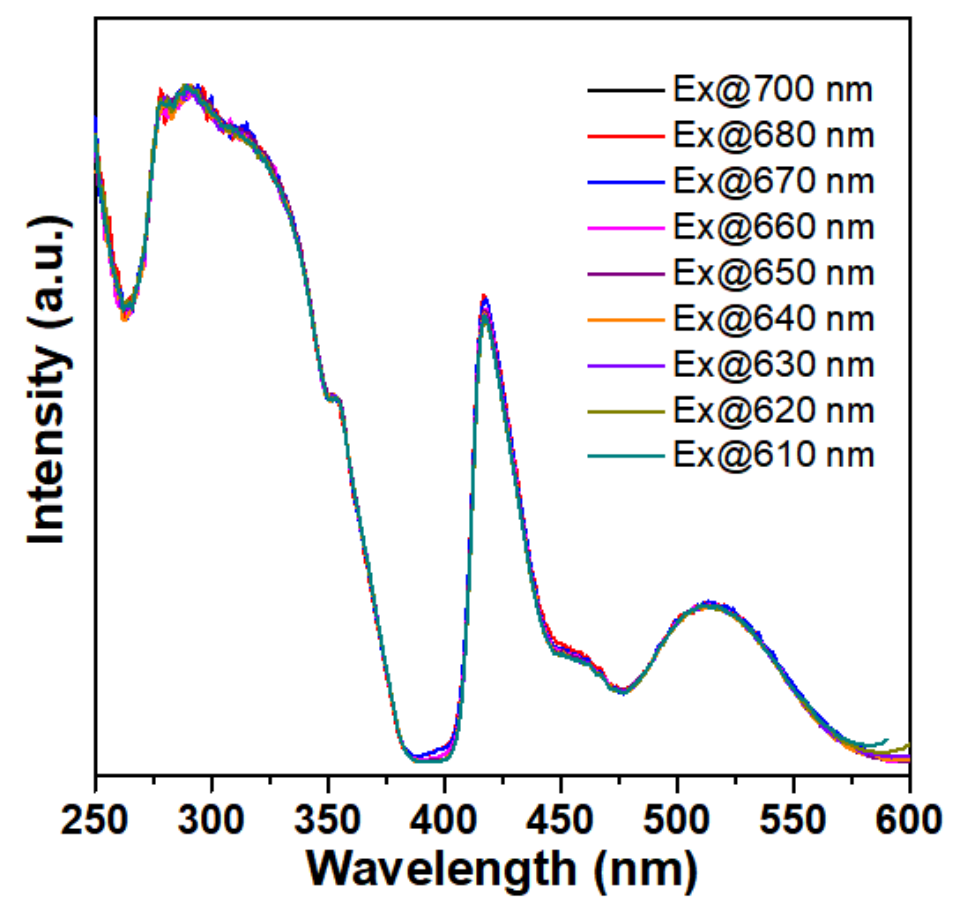

Figure S3. PLE spectra of $(4 \mathrm{AMP})_{4} \mathrm{ClMn}_{3} \mathrm{Cl}_{13} \cdot \mathrm{HCl}$ monitored at different wavelengths.

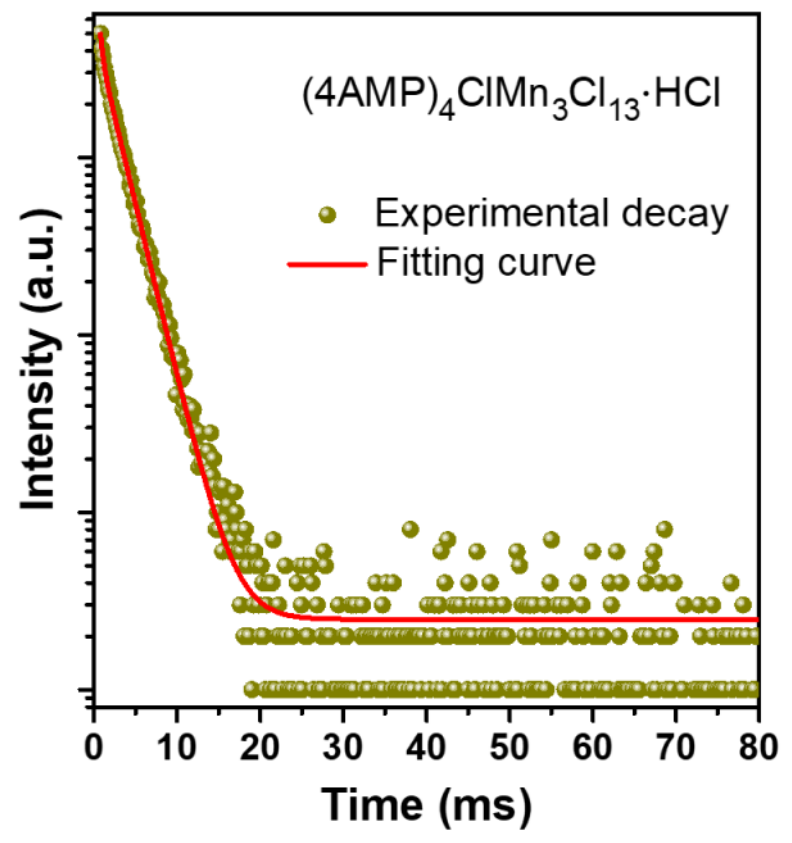

Figure S4. PL lifetime of $(4 \mathrm{AMP})_{4} \mathrm{ClMn}_{3} \mathrm{Cl}_{13} \cdot \mathrm{HCl}$ monitored at $628 \mathrm{~nm}$. 

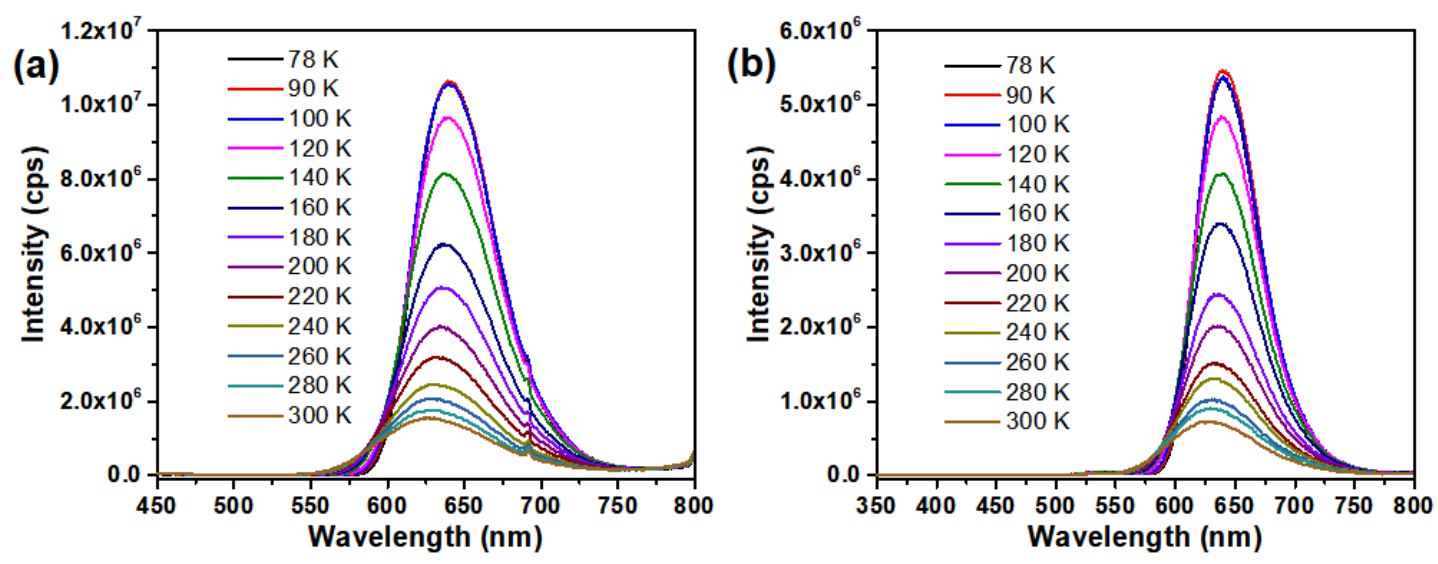

Figure S5. Temperature-dependent PL spectra of $(4 \mathrm{AMP})_{4} \mathrm{ClMn}_{3} \mathrm{Cl}_{13} \cdot \mathrm{HCl}$ upon the excitation wavelengths of $260 \mathrm{~nm}(\mathrm{a})$ and $290 \mathrm{~nm}(\mathrm{~b})$.

(a)

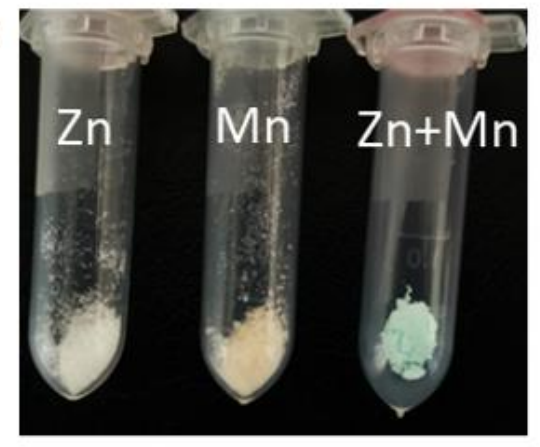

(b)

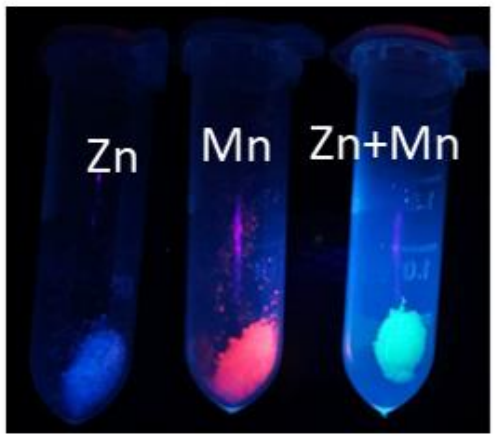

Figure S6. Photographs of compounds (4AMP) $\mathrm{ZnCl}_{4}\left(\mathbf{Z n}\right.$, left), (4AMP) ${ }_{4} \mathrm{ClMn}_{3} \mathrm{Cl}_{13} \cdot \mathrm{HCl}(\mathbf{M n}$, middle), and (4AMP) $\mathrm{Zn}_{1-\mathrm{x}} \mathrm{Mn}_{\mathrm{x}} \mathrm{Cl}_{4}(\mathbf{Z n}+\mathbf{M n}$, right) under natural light (a)and UV light (b). 


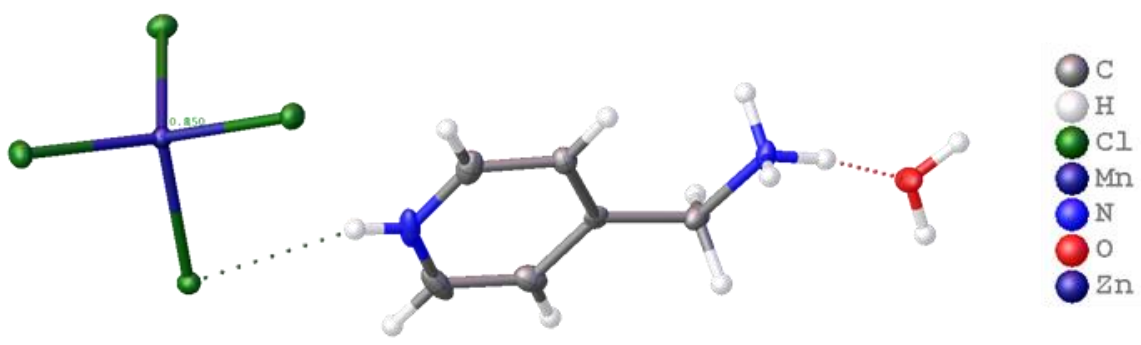

Figure S7. Crystal structure of (4AMP) $\mathrm{Zn}_{1-x} \mathrm{Mn}_{\mathrm{x}} \mathrm{Cl}_{4}$, showing the hydrogen bond between organic cations with water molecules.

(a)

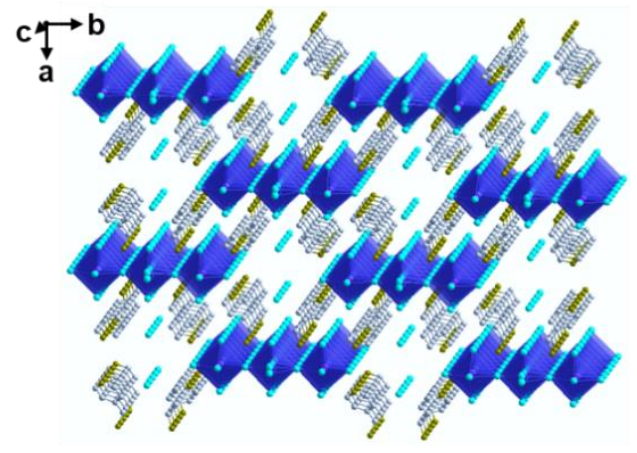

(c)

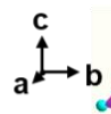

(b)

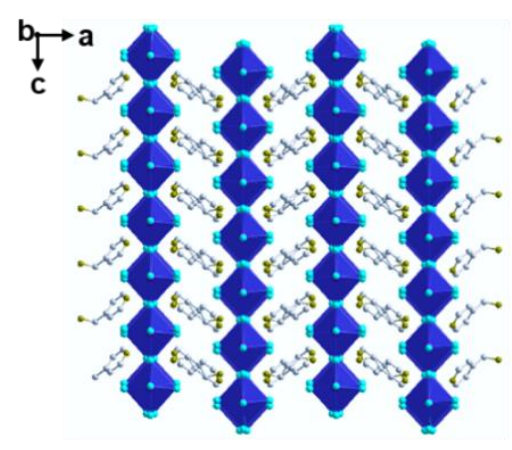

(d)

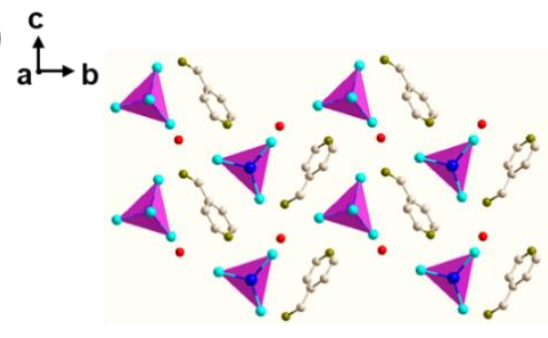

Figure S8. (a, b) Crystal structures of 1D metal halides, showing octahedral building blocks corner-sharing to form 1D chains. (c, d) Crystal structures of $0 \mathrm{D}$ metal halides, showing the tetrahedral $\left[\mathrm{MX}_{4}\right]^{2-}$ unites fully isolated by organic cations. 


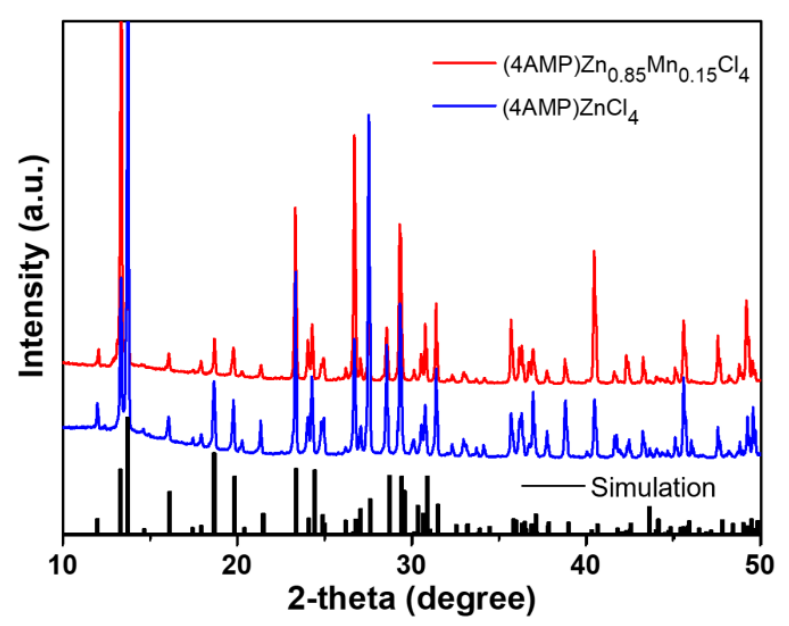

Figure S9. Comparisons between the detected and simulated XRD patterns of compound (4AMP) $\mathrm{Zn}_{0.85} \mathrm{Mn}_{0.15} \mathrm{Cl}_{4}$ (red line) and (4AMP) $\mathrm{ZnCl}_{4}$ (blue line). The simulation pattern is extracted from SCXRD of (4AMP) $\mathrm{Zn}_{0.85} \mathrm{Mn}_{0.15} \mathrm{Cl}_{4}$. 
Table S2. Measured compositions of the as-prepared single crystals by ICP-MS.

\begin{tabular}{|c|c|c|c|}
\hline Single crystals & Atoms & Precursor ratio & ICP-MS \\
\hline$(4 \mathrm{AMP}) \mathrm{Zn}_{\mathrm{x}} \mathrm{Mn}_{1-\mathrm{x}} \mathrm{Cl}_{4}$ & $\mathrm{Zn}: \mathrm{Mn}$ & $1: 2$ & $6: 1$ \\
\hline$(4 \mathrm{AMP}) \mathrm{Zn}_{\mathrm{x}} \mathrm{Mn}_{1-\mathrm{x}} \mathrm{Cl}_{4}$ & $\mathrm{Zn}: \mathrm{Mn}$ & $1: 1$ & $9: 1$ \\
\hline $\begin{array}{c}(4 \mathrm{AMP})_{4} \mathrm{ClCo}_{\mathrm{x}} \mathrm{Mn}_{3-} \\
{ }_{\mathrm{x}} \mathrm{Cl}_{13} \cdot \mathrm{HCl}\end{array}$ & $\mathrm{Co}: \mathrm{Mn}$ & $1: 2$ & $1: 13$ \\
\hline$(4 \mathrm{AMP}) \mathrm{Cox}_{\mathrm{x}} \mathrm{Mn}_{1-\mathrm{x}} \mathrm{Cl}_{4}$ & $\mathrm{Co}: \mathrm{Mn}$ & $2: 1$ & $19: 1$ \\
\hline $\begin{array}{c}(4 \mathrm{AMP})_{4} \mathrm{ClNi}_{\mathrm{x}} \mathrm{Mn}_{3-} \\
{ }_{\mathrm{x}} \mathrm{Cl}_{13} \cdot \mathrm{HCl}\end{array}$ & $\mathrm{Ni}: \mathrm{Mn}$ & $1: 4$ & $1: 100$ \\
\hline $\begin{array}{c}\left(4 \mathrm{AMP}_{4} \mathrm{ClNi}_{\mathrm{x}} \mathrm{Mn}_{3}-\right. \\
{ }_{\mathrm{x}} \mathrm{Cl}_{13} \cdot \mathrm{HCl}\end{array}$ & $\mathrm{Ni}: \mathrm{Mn}$ & $1: 2$ & $1: 50$ \\
\hline $\begin{array}{c}(4 \mathrm{AMP})_{4} \mathrm{CINi} \mathrm{Mn}_{3}- \\
{ }_{\mathrm{x}} \mathrm{Cl}_{13} \cdot \mathrm{HCl}\end{array}$ & $\mathrm{Ni}: \mathrm{Mn}$ & $3: 1$ & $1: 23$ \\
\hline $\begin{array}{c}(4 \mathrm{AMP})_{4} \mathrm{ClCu}_{x} \mathrm{Mn}_{3-} \\
{ }_{\mathrm{x}} \mathrm{Cl}_{13} \cdot 2 \mathrm{HCl}\end{array}$ & $\mathrm{Cu}: \mathrm{Mn}$ & $1: 2$ & $1.25: 1$ \\
\hline $\begin{array}{c}(4 \mathrm{AMP})_{4} \mathrm{ClCu}_{\mathrm{x}} \mathrm{Mn}_{3-} \\
\mathrm{xCl}_{13} \cdot 2 \mathrm{HCl}\end{array}$ & $\mathrm{Cu}: \mathrm{Mn}$ & $1: 1$ & $4: 1$ \\
\hline$(4 \mathrm{AMP}) \mathrm{Cu}_{\mathrm{x}} \mathrm{Mn}_{1-\mathrm{x}} \mathrm{Cl}_{4}$ & $\mathrm{Cu}: \mathrm{Mn}$ & $3: 1$ & $20: 1$ \\
\hline$(4 \mathrm{AMP})_{2}\left[\mathrm{FeCl}_{4}\right]\left[\mathrm{MnCl}_{5}\right]$ & $\mathrm{Fe}: \mathrm{Mn}$ & $1: 2$ & $1: 1$ \\
\hline$(4 \mathrm{AMP})_{2}\left[\mathrm{FeCl}_{4}\right]\left[\mathrm{MnCl}_{5}\right]$ & $\mathrm{Fe}: \mathrm{Mn}$ & $1: 1$ & $4: 1$ \\
\hline
\end{tabular}



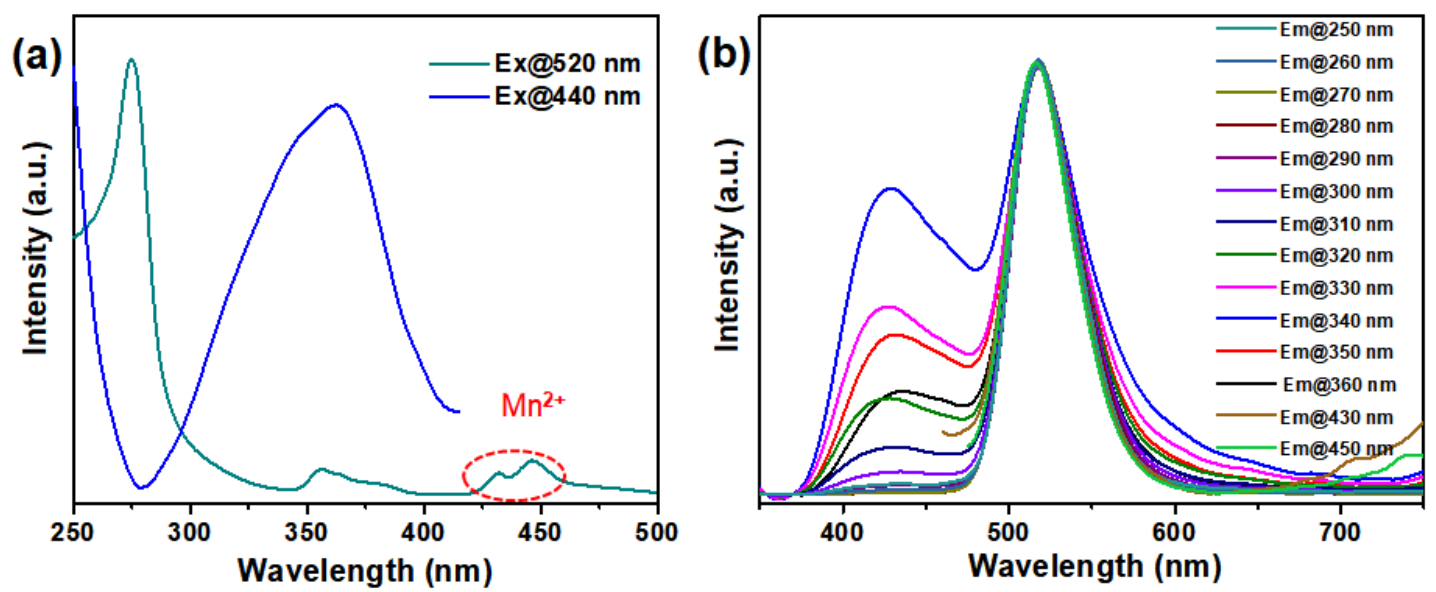

Figure S10. (a) PLE spectra of (4AMP) $\mathrm{Zn}_{0.85} \mathrm{Mn}_{0.15} \mathrm{Cl}_{4}$ monitored at $520 \mathrm{~nm}$ (green line) and 440 $\mathrm{nm}$ (blue line). The peaks in the range of 400-500 nm show the G-term transitions of $\mathrm{Mn}^{2+}$. (b) PL spectra of (4AMP) $\mathrm{Zn}_{0.85} \mathrm{Mn}_{0.15} \mathrm{Cl}_{4}$ monitored at different wavelengths.
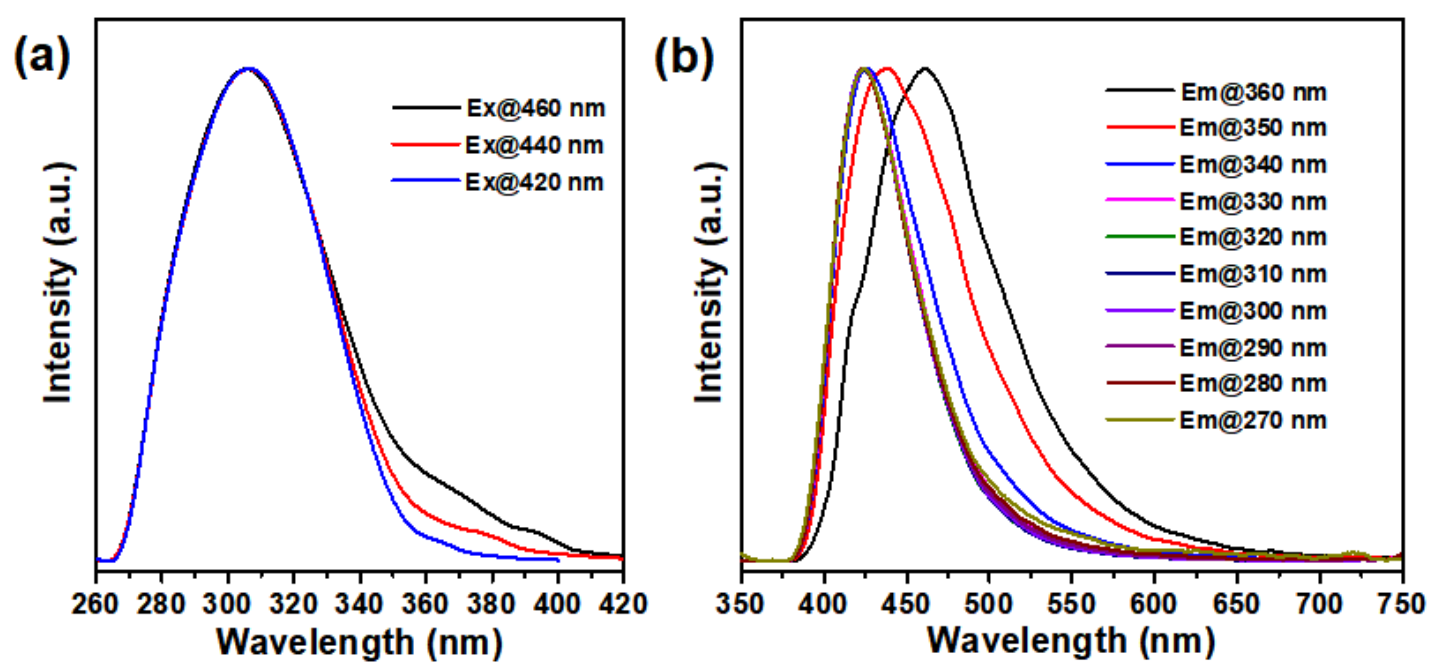

Figure S11. (a) PLE spectra of 4AMP monitored at different wavelengths. (b) PL spectra of 4AMP monitored at different wavelengths. 

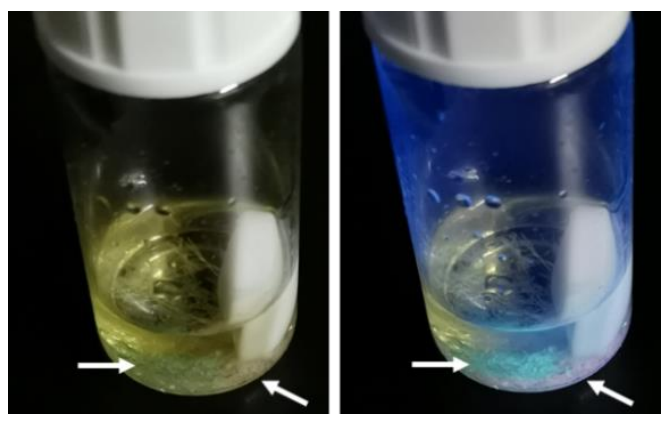

Figure S12. Photographs of the formation of mixture of 1D and OD metal halides at a low Zn: Mn ratio under natural light (left) and UV light (right).

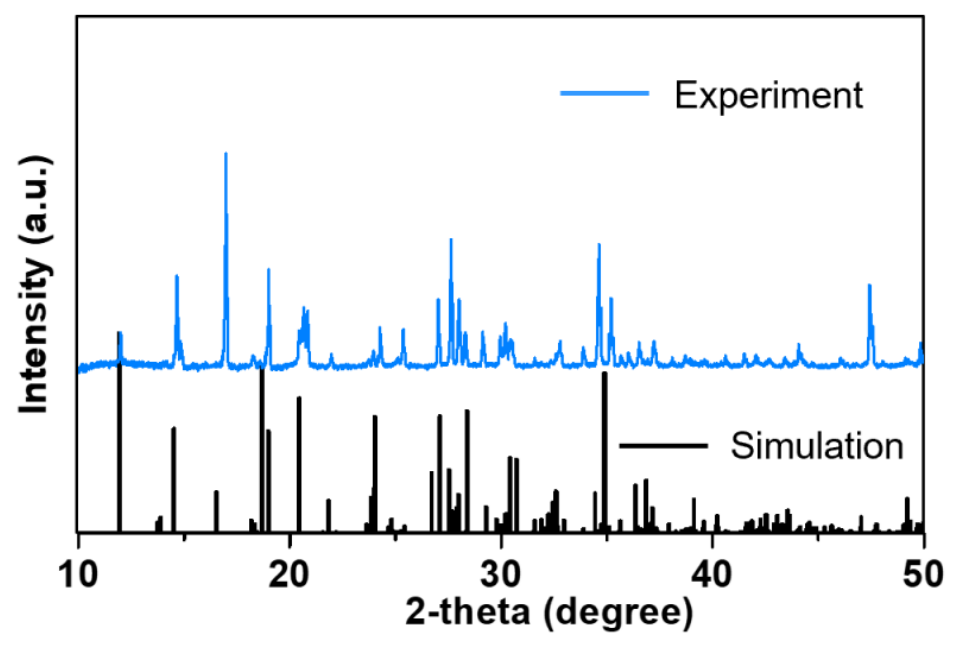

Figure S13. Comparison between the detected and simulated XRD patterns of compound $(4 \mathrm{AMP})_{2}\left[\mathrm{FeCl}_{4}\right]\left[\mathrm{MnCl}_{5}\right]$. The simulation pattern is extracted from SCXRD. 


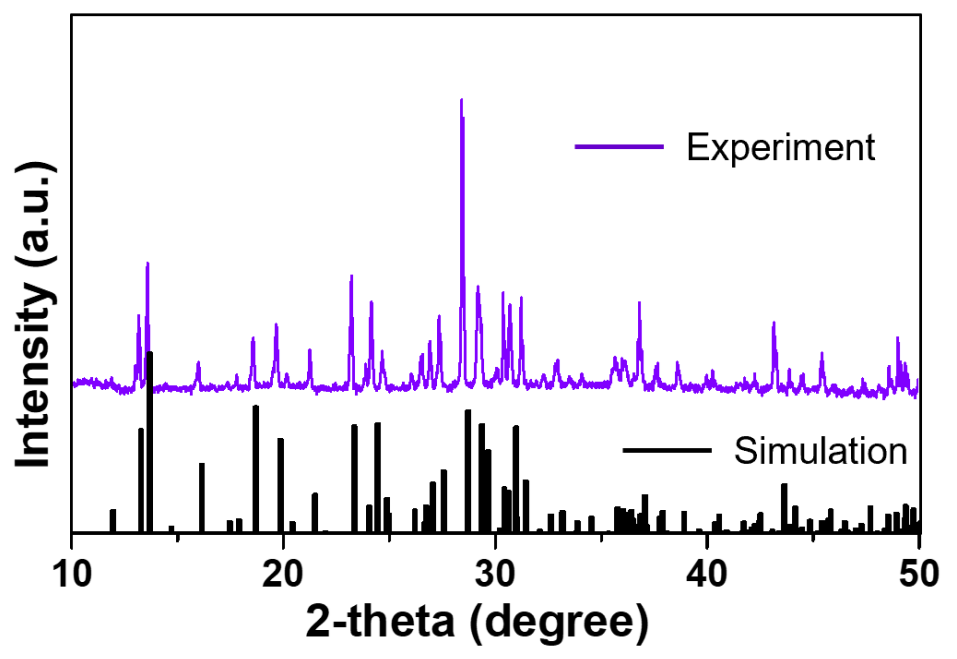

Figure S14. Comparison between the detected and simulated XRD patterns of compound (4AMP) $)_{4} \mathrm{ClCo}_{0.21} \mathrm{Mn}_{2.79} \mathrm{Cl}_{13} \cdot \mathrm{HCl}$. The simulation pattern is extracted from SCXRD.

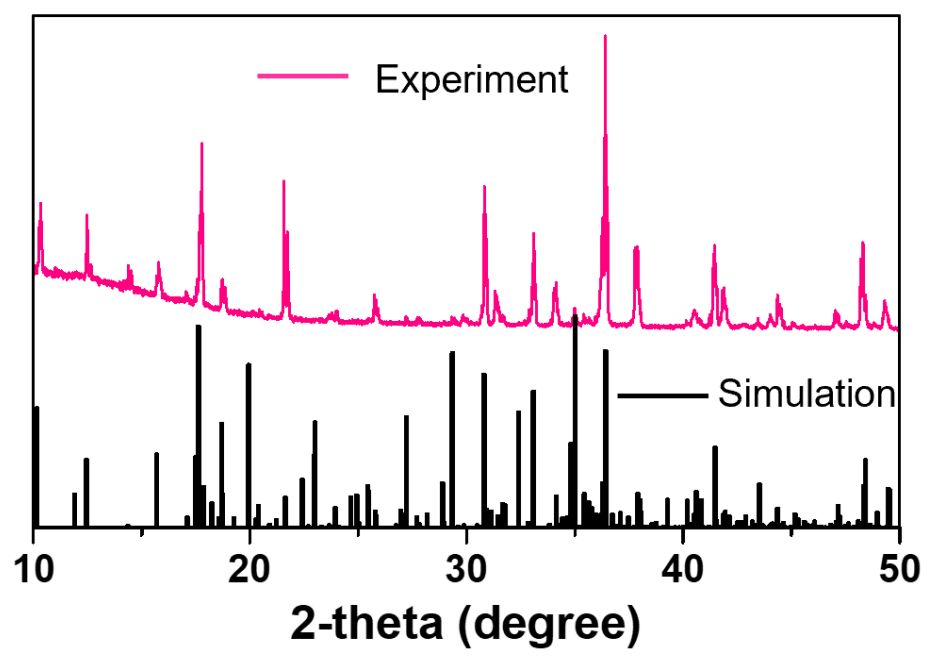

Figure S15. Comparison between the detected and simulated XRD patterns of compound (4AMP) $)_{4} \mathrm{ClNi}_{0.06} \mathrm{Mn}_{2.94} \mathrm{Cl}_{13} \cdot \mathrm{HCl}$. The simulation pattern is extracted from SCXRD. 


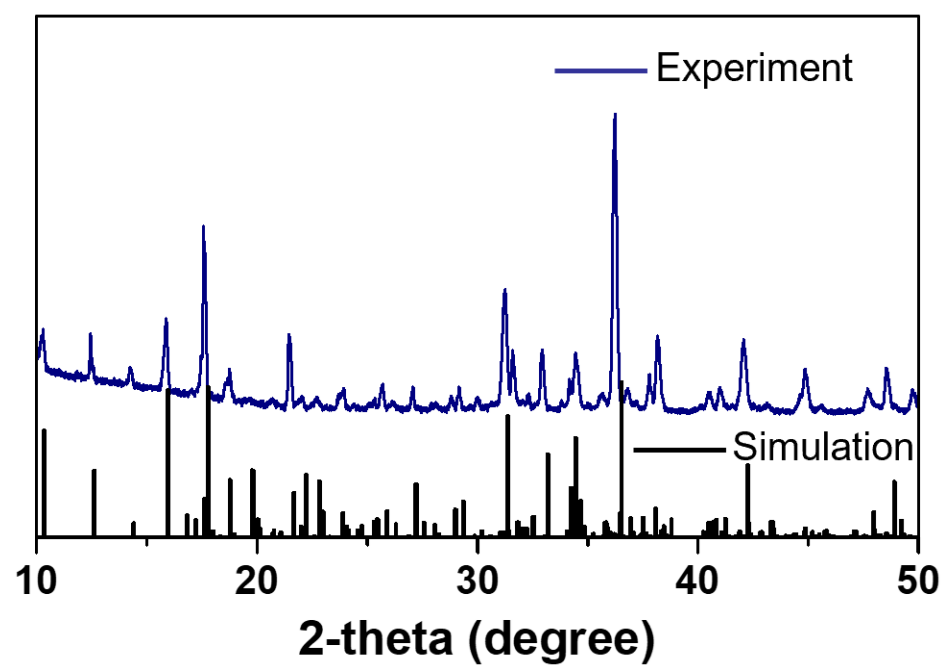

Figure S16. Comparison between the detected and simulated XRD patterns of compound (4AMP) ${ }_{4} \mathrm{ClCu}_{2.4} \mathrm{Mn}_{0.6} \mathrm{Cl}_{13} \cdot 2 \mathrm{HCl}$. The simulation pattern is extracted from SCXRD.

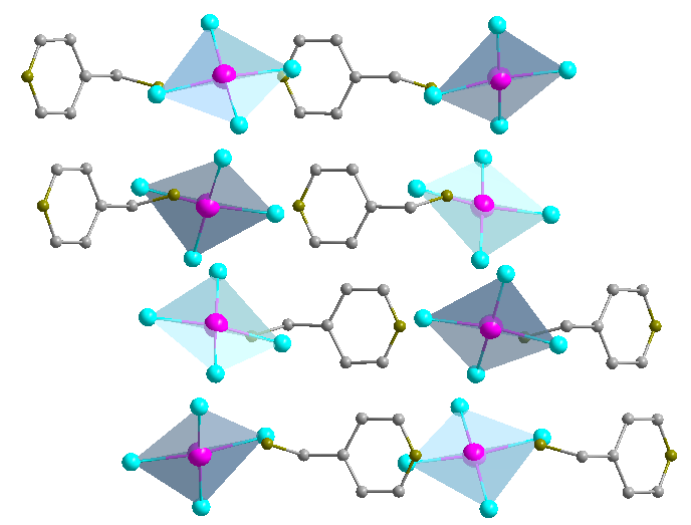

Figure S17. Crystal structure of OD (4AMP) $\mathrm{Cu}_{0.95} \mathrm{Mn}_{0.05} \mathrm{Cl}_{4}$. Magenta spheres: $\mathrm{Mn} / \mathrm{Cu}$ atoms; cyan spheres: $\mathrm{Cl}$ atoms; gray spheres: $\mathrm{C}$ atoms; dark yellow spheres: $\mathrm{N}$ atoms; light cyan polyhedra: $\left[\mathrm{Cu}_{0.95} \mathrm{Mn}_{0.05} \mathrm{Cl}_{4}{ }^{2-}\right]$ tetrahedra; $\mathrm{H}$ atoms are hidden for clarity. 


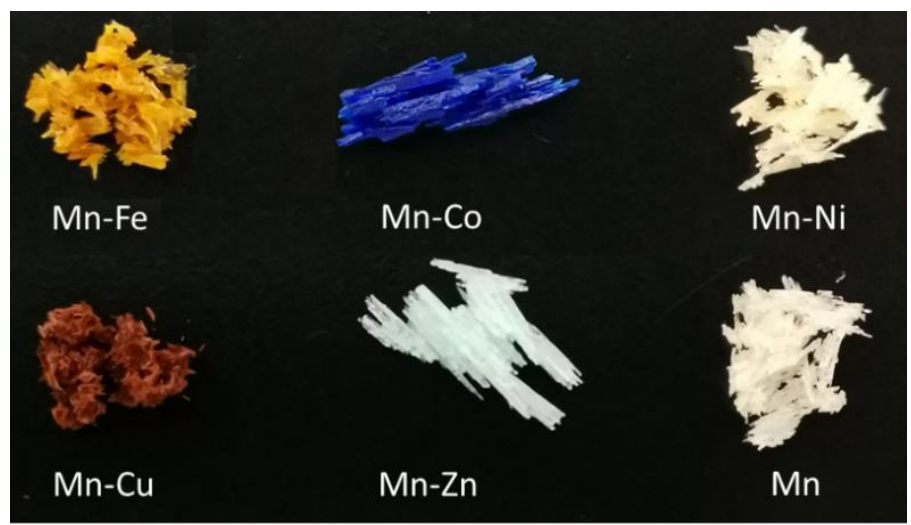

Figure S18. Photographs of the obtained single crystals under natural light.

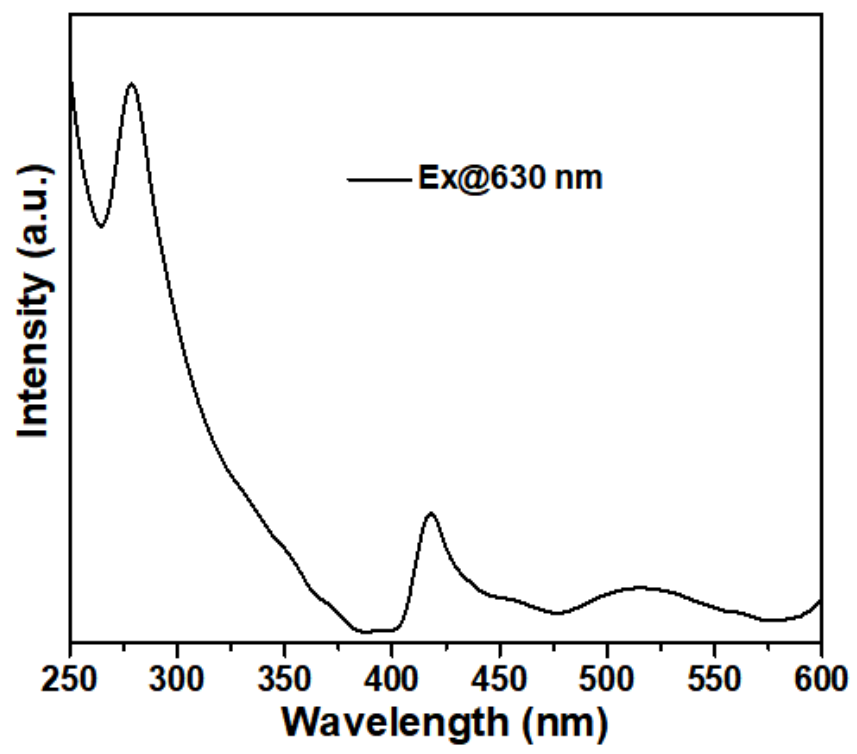

Figure S19. PLE spectrum of $(4 \mathrm{AMP})_{4} \mathrm{ClNi}_{0.06} \mathrm{Mn}_{2.94} \mathrm{Cl}_{13} \cdot \mathrm{HCl}$ monitored at $630 \mathrm{~nm}$. 


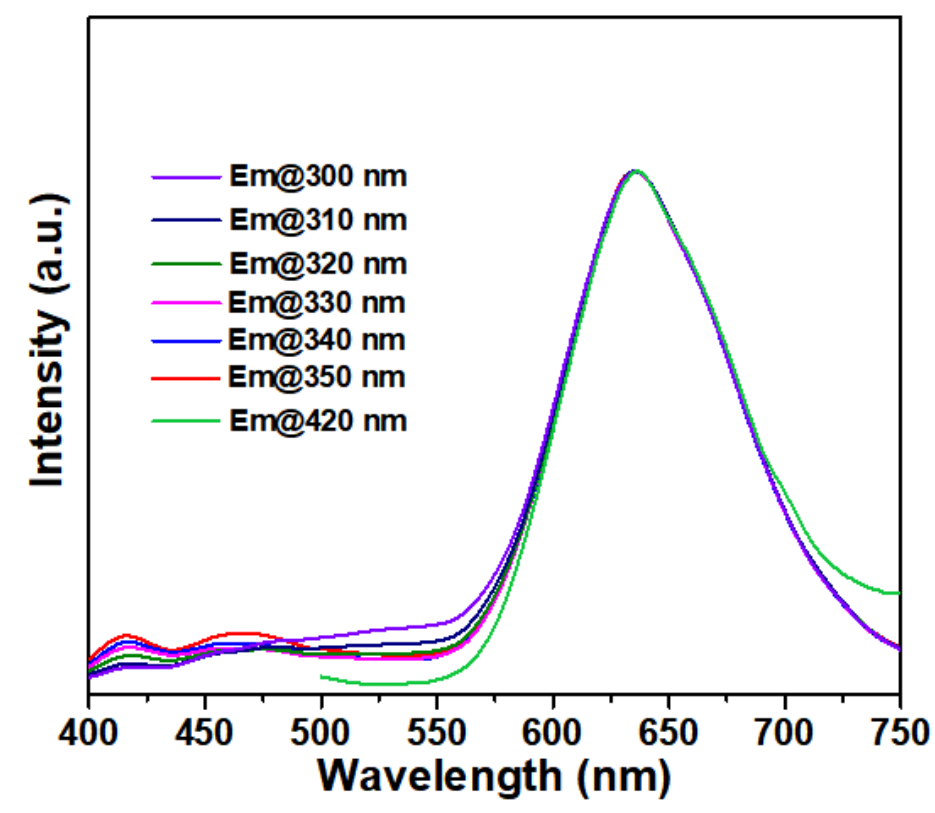

Figure S20. PL spectra of $(4 \mathrm{AMP})_{4} \mathrm{ClNi}_{0.03} \mathrm{Mn}_{2.97} \mathrm{Cl}_{13} \cdot \mathrm{HCl}$ upon the excitation at various wavelengths. 